Author running head: F. De Biasio et al.

Title running head: $O B P$ expression patterns in the pea aphid

Correspondance: Patrizia Falabella, Dipartimento di Scienze, Università della Basilicata, via dell'Ateneo Lucano 10, 85100 Potenza, Italy. Tel +39 0971205501; fax +39 0971205503; email:patrizia.falabella@unibas.it

ORIGINAL ARTICLE

\title{
Expression pattern analysis of odorant-binding proteins in the pea aphid
}

\section{Acyrthosiphon pisum}

Filomena De Biasio ${ }^{1}$, Lea Riviello ${ }^{1}$, Daniele Bruno $^{2}$, Annalisa Grimaldi², Terenzio Congiu $^{3}$, Yu Feng Sun ${ }^{4}$ and Patrizia Falabella ${ }^{1}$

${ }^{1}$ Università della Basilicata, Dipartimento di Scienze, via dell'Ateneo Lucano 10, 85100 Potenza, Italy, ${ }^{2}$ Università dell'Insubria, Dipartimento di Biotecnologie e Scienze della Vita, via J. H. Dunant 3, 21100 Varese, Italy, ${ }^{3}$ Università dell'Insubria, Dipartimento di Scienze Chirurgiche e Morfologiche, via Monte Generoso 3, 21100 Varese, Italy and ${ }^{4}$ Chinese Academy of Agricultural Sciences, Institute of Plant Protection, Beijing, China

This is an Accepted Article that has been peer-reviewed and approved for publication in the Insect Science but has yet to undergo copy-editing and proof correction. Please cite this article as doi: 10.1111/1744-7917.12118. 


\begin{abstract}
Odorant-binding proteins (OBPs) are soluble proteins mediating chemoreception in insects. In previous research, we investigated the molecular mechanisms adopted by aphids to detect the alarm pheromone $(E)-\beta$-farnesene and we found that the recognition of this and structurally related molecules is mediated by OBP3 and OBP7. Here we show the differential expression patterns of five selected OBPs (OBP1, OBP3, OBP6, OBP7, OBP8) obtained performing quantitative RT-PCR and immunolocalization experiments in different body parts of adults and in the five developmental instars, including winged and unwinged morphs, of the pea aphid Acyrthosiphon pisum. The results provide an overall picture that allows us to speculate on the relationship between the differential expression of OBPs and their putative function. The expression of OBP3, OBP6 and OBP7 in the antennal sensilla suggests a chemosensory function for these proteins, whereas the constant expression level of OBP8 in all instars could suggest a conserved role. Moreover OBP1 and OBP3 are also expressed in non-sensory organs. A light and scanning electron microscopy study of sensilla on different body parts of aphid, in particular antennae, legs, mouthparts and cornicles-cauda, completes this research providing a guide to facilitate the mapping of OBP expression profiles.
\end{abstract}

Key words aphids, chemosensilla, immunohistochemistry, microscopy, odorant-binding protein (OBP) 


\section{Introduction}

Olfactory and gustatory systems play crucial roles in insect behaviors, such as in locating food, choosing oviposition sites and mating. The chemosensory system of insects can recognize and discriminate many different chemicals through chemosensilla, specialized cuticular structures innervated by sensory neurons, that send axons to the antennal lobes in the brain for stimulus processing (Hansson, 1999; Hansson et al., 2003; Hildebrand, 1996).

Chemosensilla come in a variety of shapes and present on their surface one (uniporous sensilla) or several pores (multiporous sensilla) that allow access of semiochemicals, most of which are hydrophobic molecules, into the sensillar lymph, an aqueous medium that bathes the dendrites of sensory neurons.

The sensillum lymph is characterized by a high concentration of small soluble proteins, the odorant-binding proteins (OBPs) (Pelosi et al., 2006; Vogt, 2003). In recent years, these polypeptides, first regarded as passive carriers for pheromones and odorants through the lymph hydrophilic barrier, have been recognized as very important elements in the process of chemodetection and olfactory recognition. It has been shown that a mutant of LUSH, one of the OBPs of Drosophila melanogaster required for perception of the male pheromone vaccenyl acetate (Xu et al., 2005), can activate the olfactory neuron even in the absence of the pheromone, since this mutant can mimic the conformation of LUSH when bound to vaccenyl acetate (Laughlin et al., 2008). Working with some species of Drosophila, Matsuo et al. (2007) showed that the attraction or repellency to two fatty acids can be modified by switching the genes encoding two OBPs between different Drosophila species. Another study analyzed the response to several odorants of 17 strains of $D$. melanogaster, each deficient in one specific OBP. Fruit flies of different strains exhibited different olfactory responses to the same odorants, suggesting that OBPs are involved in odor recognition and discrimination (Swarup et al., 2011). In aphids, the repellency to the alarm pheromone (E)- $\beta$-farnesene and 
related compounds is linked to their affinity to OBP3 and/or OBP7 (Sun et al., 2012a), further supporting the idea that these proteins are involved in decoding the chemical information of odorants and pheromones.

Acyrthosiphon pisum (Harris) (Homoptera, Aphididae), commonly known as the pea aphid, is a pest for many different kinds of leguminous plants (Blackman \& Eastop, 2007). Like other aphid species, it causes damage to the host plant either directly by feeding on their phloem sap, or indirectly by transmitting viruses (van Emden \& Harrington, 2007). Like all insects, aphids rely on their chemical sensing for different essential tasks in their life, such as host location, mate selection, detection of danger (van Emden \& Harrington, 2007), and therefore strategies interfering with their chemical communication can be applied to population control (Dewhirst et al., 2010; Sun et al., 2011, 2012a; Zhou et al., 2010a; Zhu et $a l ., 2006)$. The understanding of insect chemosensory pathway can be exploited to modify the pest behavior optimizing the use of semiochemicals in monitoring and controlling strategies in sustainable agriculture (Plettner, 2002; Sun et al., 2012a). The characterization of OBP expression profiles in different developmental stages and various tissues could provide essential information to plan, in a focused way, further functional assays, obtaining a greater understanding of the insect chemosensory system. The recent sequencing of the pea aphid genome allowed the identification and annotation of 15 genes encoding OBPs (Zhou et al., 2010b). Here we analyze, by quantitative RT-PCR and immunolocalization experiments, the expression profiles of five OBPs (OBP1, OBP3, OBP6, OBP7, OBP8), a consistent part of aphid repertoire of these polypeptides, in different body parts (especially the chemosensory organs) and in the five instars of A. pisum, including winged and unwinged adults. A light and electron microscopy study of sensilla on different body parts of aphid, in particular antennae, legs, mouthparts and cornicles-cauda, completes this research providing a guide to facilitate the mapping of OBP expression profiles. Our results allow us to gain clues about the This article is protected by copyright. All rights reserved. 
relation between the A. pisum OBP expression patterns and their functional implications, mainly in chemoreception.

\section{Materials and methods}

\section{Insect rearing and sample collection}

Acyrthosiphon pisum was reared on potted broad bean plants (Vicia faba L) at $20 \pm 1{ }^{\circ} \mathrm{C}$, $75 \% \pm 5 \% \mathrm{RH}$ and $18 \mathrm{~h}$ photoperiod. Aphid cultures were started with insect material originally collected from alfalfa plants, in Southern Italy (Eboli, SA). In order to synchronize aphid samples, new born aphids obtained by parthenogenetic females were separated from their mother removing her from the plant. New born aphids were maintained on plants for 6 days and individuals were collected at 5 different development stages from first instar to unwinged (apterous) adults. Winged aphids (alatae) were collected occasionally from the colony. Samples were flashed frozen with liquid nitrogen and stored at $-80{ }^{\circ} \mathrm{C}$ until RNA extraction. Different body parts (antennae, deantennaed head, legs, cornicles-cauda and remaining body part) from adult apterous females were dissected under the microscope, fixed and immediately processed for light and scanning electron microscopy, immunolocalization experiments or homogenized in TRI Reagent (Sigma) and stored at $-80{ }^{\circ} \mathrm{C}$ until RNA extraction.

\section{Light microscopy}

Samples were fixed for $2 \mathrm{~h}$ in $0.1 \mathrm{~mol} / \mathrm{L}$ cacodylate buffer, $\mathrm{pH} \mathrm{7.2,} \mathrm{containing} 2 \%$ glutaraldehyde and $4 \%$ paraformaldehyde. Specimens were then washed in the same buffer and post-fixed for $2 \mathrm{~h}$ with $1 \%$ osmium tetroxide in cacodylate buffer, $\mathrm{pH}$ 7.2. After standard 
serial ethanol dehydration (70\%-100\%), samples were embedded in an Epon-Araldite 812 mixture (Sigma). Sections were obtained with a Reichert Ultracut S ultratome (Leica). Semithin sections $(700 \mathrm{~nm})$ were stained by conventional methods (crystal violet and basic fuchsin) according to reference (Moore et al., 1960) and subsequently observed under a light microscope (Olympus). Images were acquired with a Nikon D5-5M camera.

\section{Scanning electron microscopy (SEM)}

Adult apterous aphids were processed as already described by Sun et al. (2013). They were fixed for $2 \mathrm{~h}$ in $70 \%$ ethanol and then cleaned in ultrasonic bath for $1 \mathrm{~min}$ in the same solution. Subsequently samples were dehydrated $100 \%$ ethanol for $30 \mathrm{~min}$, dried in air, coated in gold by K250 sputter coater (Emitech) and examined with SEM-FEG XL-30 microscope (Philips).

Total RNA extraction and cDNA synthesis

Total RNA was extracted using TRI Reagent (Sigma), following the manufacturer's instructions. The RNA concentration was measured by optical density, at $260 \mathrm{~nm}$, using a NanoDrop ND-1000 spectrophotometer (Nanodrop Technologies). The purity of RNA was assessed at an absorbance ratio of OD260/280 and OD260/230 and the integrity was checked with $0.8 \%$ agarose gel electrophoresis. For an efficient removal of the genomic DNA contamination, the samples were treated with $1 \mathrm{U}$ of DNase I (Deoxyribonuclease I, Amplification Grade, Invitrogen-Life Technologies) per $\mu \mathrm{g}$ of RNA for $15 \mathrm{~min}$ at room temperature. The reaction was stopped by the addition of $1 \mu \mathrm{L}$ of $25 \mathrm{mmol} / \mathrm{L}$ EDTA and incubation at $65{ }^{\circ} \mathrm{C}$ for 10 min. cDNA was synthesized with the SuperScript ${ }^{\circledR}$ III First-Strand Synthesis System for RT-PCR (Invitrogen-Life Technologies), according to the 
manufacturer's protocol, using $500 \mathrm{ng}$ of total RNA per sample. The synthesized cDNA was stored at $-20^{\circ} \mathrm{C}$ until use.

\section{Quantitative RT-PCR ( $q R T-P C R)$}

Quantitative RT-PCR experiments were carried out in a 7500 Fast Real-Time PCR System (Applied Biosystems-Life Technologies), on cDNA samples prepared from five instars, including winged and unwinged adults, and different body parts (antennae, deantennaed head, legs, cornicles-cauda and remaining body part) of unwinged adults, as described above, following the guidelines reported in Minimum Information Required for Publication of Quantitative Real-Time PCR experiments (MIQE) (Bustin et al., 2009).

Actin (NCBI Reference Sequence: NM_001142636.1), a pea aphid constitutive gene, was chosen as the reference gene for normalization. Specific primers were designed for each $A$. pisum OBP gene (OBP1, OBP3, OBP6, OBP7, OBP8) and the reference gene, using Primer Express v3.0 software (Applied Biosystems-Life Technologies) (Table 1). OBP gene sequences were obtained from the NCBI Nucleotide Database (http://www.ncbi.nlm.nih.gov/nuccore/).

PCR amplification was performed using GoTaq qPCR Master Mix (Promega). The reactions were carried out in a $20 \mu \mathrm{L}$ final volume containing $5 \mu \mathrm{L}$ cDNA solution $(60 \mathrm{ng} / \mu \mathrm{L})$ and $0.3 \mu \mathrm{mol} / \mathrm{L}$ primer final concentration. Cycling conditions for all genes were: 2 min at 95 ${ }^{\circ} \mathrm{C}$, followed by 40 cycles of $15 \mathrm{~s}$ at $95{ }^{\circ} \mathrm{C}$ and 1 min at $60{ }^{\circ} \mathrm{C}$. At the end of each run a melting curve analysis was performed to confirm the specificity of PCR products. All amplification reactions were run in triplicate (technical replicates) and included negative controls (non-template reactions, replacing cDNA with $\mathrm{H}_{2} \mathrm{O}$ ). All the experiments were performed for a set of three biological replicates. 
To evaluate the gene expression levels, relative quantification was performed using equations described by Liu and Saint (2002), based on PCR amplification efficiencies of reference and target genes. Amplification efficiency of each target gene and Actin was determined according to the equation $\mathrm{E}=10^{(-1 / \mathrm{S})}-1$ (Lee et al., 2006), where $\mathrm{S}$ is the slope of the standard curve generated from four serial 10-fold dilutions of cDNA. All data (mean \pm SE) were compared with analysis of variance (ANOVA) and Tukey's HSD test using R software version 2.10.2 (Development Core Team, 2009).

\section{Whole mount immunolocalization experiments}

Different body parts (antennae, deantennaed head, legs, cornicles-cauda and remaining body part) from adult apterous aphids were dissected under the microscope and washed twice with phosphate buffered saline (PBS), $\mathrm{pH}$ 7.4. After washing, samples were fixed for $2 \mathrm{~h}$ in 4\% paraformaldehyde in PBS for 40 min and then washed twice with the same buffer. Subsequently samples were incubated for 30 min with PBS, containing $2 \%$ BSA and $0.1 \%$

Tween and incubated for $1 \mathrm{~h}$ at room temperature with polyclonal antibodies raised in rabbit against each different OBPs (kindly provided by Prof. Pelosi, University of Pisa), diluted 1:200. Samples, washed with PBS several times, were incubated for $1 \mathrm{~h}$ in a dark moist chamber with the suitable secondary antibodies conjugated with tetramethylrhodamine (TRITC) (Jackson, Immuno Research Laboratories), diluted 1:200. Controls were carried out omitting the incubation with the primary antibody. Coverslips were mounted with Cityfluor (Cityfluor Ltd) and samples examined under a confocal laser microscope Leica TCS SP5 (laser set at $568 \mathrm{~nm}$ for rhodamine). 


\section{Results}

\section{Light and scanning electron microscopy (SEM) of sensilla}

Light and SEM microscopy observations in our study would represent a guide to facilitate the mapping of OBP expression profiles.

Microscopy examination of adult aphid legs and antennae showed differences in the morphology and distribution of sensilla (Fig. 1A-C; Fig. 2A-L). Legs presented numerous trichoid sensilla uniform in size, shape and distribution (Fig. 1A, B), inserted in the leg wall through a well-defined socket and equipped with a thin cuticular sheath (Fig. 1C).

On the entire antenna surface the trichoid sensilla were present at a lower density than in the leg (Fig. 2A). On A. pisum antennae we observed both types of trichoid sensilla previously described in aphids (Bromeley et al., 1980; Shambaugh et al., 1978). Type II was mainly found on the antennal tip and along the processus terminalis (5th and 6th segments) (Fig. 2A, B). They appeared as short hairs with a blunt tip and a single apical pore (Fig. 2AC). Type I occurred along the whole length of antennae and showed a slightly swollen poreless tip (Fig. 2J, K). We also confirmed the presence of the primary rhinaria on the 5th and 6th antennal segments (Fig. 2D-I) previously described in A. pisum by Shambaugh et al. (1978). A large placoid sensillum near to the distal end of the fifth segment (Fig. 2H, I) and on the sixth segment one large placoid sensilla, two smaller ones (Fig. 2D, E) and four coeloconic pegs (Fig. 2D, F, G) were visible. A cuticular fringe surrounded all sensilla of primary rhinaria (Fig. 2D-I). The placoid sensilla were circular plates, whereas the coeloconic sensilla appeared as pegs in a cavity (Fig. 2D-I). According to the shape of cuticular projections at peg tip, the coeloconic sensilla were classified as type I and type II (Fig. 2F, G). On the third antennal segment we confirmed the presence of both type I trichoid sensilla and secondary rhinaria constituted by placoid sensilla with a smooth ridge (Fig. 2JL). 
Long hairs, showing grooves on their tip (Fig. 3A, B), were observed on both side of $A$. pisum mouthpart appendages. Moreover, at the distal tip of aphid labium, 16 short peg sensilla symmetrically distributed were present (Fig. 3A, C). Although these pegs had a poreless tip, as already described by Wensler (1977) and Tjallingii (1978), at their base we observed a dilated and infolded region of about 0.2 micron (Fig. 3C), suggesting the presence of molting pore-like structures similar to those previously reported by Tjallingii (1978). Finally, SEM observation showed, besides hair-like structures (Fig. 3D), digitiform projections, arranged in groups (Fig. 3E), at the terminal part of aphid body on cauda.

OBP expression patterns in different body parts and instars of $\mathrm{A}$. pisum

To investigate the expression level of $A$. pisum OBPs (OBP1, OBP3, OBP6, OBP7, OBP8) in different body parts and instars, quantitative RT-PCR experiments were performed using gene-specific primers (Table 1) and Actin as the reference gene. The Actin expression level does not change across all treatments in our experiments. The means of Actin $\mathrm{Cq}$ values were not statistically different (ANOVA, $P=0.07233$ ).

For OBP6 and OBP7 the highest transcript levels were observed in the antennae (Fig. 4). Moreover, we found that genes encoding OBP1 and OBP3 were mainly expressed in the terminal region of the abdomen (Fig. 4), that on the upper part houses cornicles (Fig. 6B).

These results were complemented by the whole mount immunolocalization experiments of the respective proteins, using polyclonal antibodies prepared against $A$. pisum recombinant OBPs (Figs. 5, 6). These proteins are highly concentrated in the lymph surrounding the sensory dendrites of olfactory sensilla. In particular, OBP3, OBP6 and OBP7 were immunolocalized in the type II trichoid sensilla (Fig. 5A-H) and in the primary rhinaria 
located on the fifth and on the sixth segment of antenna (Fig. 5I-P). The OBP3 was expressed in the lymph of type II trichoid sensilla located on the distal region of antenna and into placoid and coeloconic sensilla (Fig. 5E, I). By contrast, the type II trichoid sensilla located on the tip of antenna, the large placoid sensillum on the 5th segment and the type I trichoid and placoid sensilla on the 3rd segment were not labeled by the antiserum against OBP3 (Fig. 5A, M, Q). OBP6 was immunolocalized in the lymph of all sensilla located on 6th, 5th and 3rd antennal segments, except in the type I trichoid sensilla and in the 6th segment coeloconic sensilla (Fig. 5B, F, J, N, R). Finally the lymph of the type II trichoid, placoid and coeloconic sensilla on the processus terminalis were labeled specifically by the antibody against OBP7, while the 3rd segment type I trichoid and placoid sensilla were not stained by this antibody (Fig. 5C, G, K, O, S). No signal was detected in control experiments (Fig. 5D, H, L, P, T).

OBP1 and OBP3 were also detected in the terminal region of the body (Fig. $6 \mathrm{~A}-\mathrm{N}$ ) in the hair-like structures (Fig. 6F, H, K), in the cornicles (Fig. 6C, D) and on the cauda digitiform projections (Fig. 6F, G, I, J).

In contrast to this, we found that the gene encoding OBP8 was mainly expressed in head (Fig. 4) and the respective protein was mainly detected in the buccal apparatus (Fig. 6O), at the base of the sixteen short pegs on the labium tip and in the inner lymph of hair-like sensilla on the labium side walls (Fig. 6P, Q). No signal was detected in control experiments (Fig. 6E, $\mathrm{L}-\mathrm{N}, \mathrm{R})$.

As far as the legs are concerned, we found a very low expression level for all the considered OBPs and a very slight signal in immunolocalization experiments (data not shown).

Transcription profiling for the OBP genes was also performed for all A. pisum developmental stages including the four pre-reproductive stages and the winged and unwinged adult morph (Fig. 7). OBP1, OBP3 and OBP7 genes showed an expression peak in This article is protected by copyright. All rights reserved. 
the fourth instar and unwinged adult stage, whereas the OBP6 transcript was mainly detected in winged adults respect to the unwinged and immature stages (Fig. 7). On the contrary OBP8 is constantly expressed in all aphid instars (Fig. 7).

\section{Discussion}

Understanding the chemical communication mechanisms in aphids provides the basic knowledge in order to develop new strategies for the biological control of these pests which are of economic importance (Zhou et al., 2010a).

In this paper, we investigated the morphology and distribution of sensilla and the expression of five OBPs (OBP1, OBP3, OBP6, OBP7, OBP8) in different body parts, instars and morphs of pea aphid suggesting a possible relationship between the OBP localization and their function.

An overall picture of A. pisum sensilla by light and scanning electron microscopy (SEM)

Insect sensilla are classified on a morphological basis in different types, such as trichoidea, coeloconica, placoidea and others. Trichoid sensilla are innervated hairs projecting out from the cuticle, the caeloconic sensillum resembles a peg recessed in a pit and the placoid type is a multiporous sensillum that consists of a thin oval plate surrounded or not by a cuticular ridge (Bromley et al., 1979, 1980; Hansson, 1999; Ryan, 2002; Shields \& Hildebrand, 2001). On aphid antenna, consisting of six segments numbered from the base and including a scape (1st), a pedicel (2nd) and four flagella (3rd-6th), two types of trichoid sensilla have been reported. Type I sensilla occur along the whole length of the antenna, whereas type II, usually 
shorter than type I, with a blunt tip, is found on the distal part of the antenna (Bromley et al., 1980; Sun et al., 2013). We confirmed the presence of both type I trichoid sensilla, distributed on the whole antenna surface, and type II trichoid sensilla, localized on the shaft of 5th and 6th segments and on the antennal tip, crowned by four blunt tipped uniporous hairs; these two types of sensilla could be involved in mechanosensing and/or in contact chemoreception (Bromley et al., 1980; Isidoro et al., 1996; Pickett et al., 1992; Romani \& Rossi Stacconi, 2009; Sun et al., 2013). A gustatory function for type II trichoid sensilla seems to be supported also by their location in the distal part of the antennae. When aphids come into contact with the plant, leaf surface characteristics and chemicals (for example hydrocarbons, waxes, fatty acids) are monitored by contact (gustatory) receptors on the antennal tips (Messchendorp et al., 1998) and tarsi (Pettersson et al., 2007).

Antennal SEM observations confirmed the distribution and morphology of primary rhinaria on the fifth and sixth segments as previously described for A. pisum by Shambaugh et al. (1978) and for other aphid species (Bromley et al., 1979; Sun et al., 2013). They are constituted by one large placoid sensillum, two smaller ones, four coeloconic pegs located on the 6th segment, and by a single large placoid sensillum on the 5th one. These sensilla are called "primary rhinaria" because they are present in all aphid life stages and morphs (Flögel, 1905). Electrophysiological recordings from these sensory areas in adult insects showed that they detect common leaf volatiles and alarm pheromone (van Giessen et al., 1994; Wohlers \& Tjallingii, 1983). Moreover, on the 3rd segment of apterous females we observed the secondary rhinaria composed by placoid sensilla morphologically similar to those described in other aphid species (Bromley et al., 1979; Shambaugh et al., 1978; Sun et al., 2013). Secondary rhinaria are more abundant in males than gynoparae and winged virginoparae and are sensitive to sex pheromone components and plant volatiles (Marsh, 1975; Pettersson, 1971; Pickett et al., 1992). 
Numerous trichoid sensilla uniform in size, shape and distribution were present on the entire leg surface at higher density than in antennae.

On the mouthpart appendages we observed hair-like structures distributed on both side of labium and sixteen short peg sensilla on the labial tip. The short pegs have been already described in other species as mechanoreceptors by Wensler (1977) and Tjallinglii (1978). Moreover, our SEM morphological observation highlighted fissure-like structures at the tip of long hair sensilla. To our knowledge this is the first description of $A$. pisum mouthpart trichoid sensilla with a grooved tip, however it deserves further investigations to confer on them a possible role in chemoreception. Finally, SEM observation showed, besides hair-like structures, digitiform projections, arranged in groups, at the terminal part of aphid body on cauda, whose function is still unknown.

Further studies are needed to better define the ultrastructure of each sensillum that could provide additional information regarding their specific function in perception processes.

OBP expression patterns in different body parts and instars of A. pisum

To better elucidate the potential role of considered OBPs into chemoreception, we provided an insight about the function of these polypeptides correlated to their expression and localization.

Quantitative RT-PCR and immunostaining experiments revealed high expression of OBP7 and OBP6 in the antennal sensilla of adults, suggesting an olfactory role for these proteins in detecting (E)- $\beta$-farnesene, plant volatiles or sex pheromone (Bromley et al., 1979; Sun et al., 2013). Aphid antenna present different types of sensilla, well documented for some aphid species (Bromley et al., 1979; Shambaugh et al., 1978; Sun et al., 2013). Our observations confirmed the morphology and the distribution of these sensilla on A. pisum antennae. 
Immunohistochemistry analysis showed the expression of OBP3, OBP6 and OBP7 in the lymph of 6th segment placoid sensilla, with a well documented olfactory function, supporting a chemosensory role for these proteins. The immunoreactivity for the antibody against OBP6 was also observed in type II trichoid sensilla and in placoid sensilla on 5th and 3th segments, while OBP7 was not immunolocalized in placoidea on 3th segment. Otherwise the anti-OBP3 antibody labeled neither sensilla on 3th segment nor those on 5th segment nor the lymph of the four trichoid sensilla located on the antennal tip. These results are in agreement with those obtained in RT-PCR experiments and could contribute to explain the different expression levels of these three OBPs in A. pisum antennae, all involved in chemosensory reception. Moreover the expression of OBP3 and OBP7 in multiporous placoid sensilla supports their involvement in the perception of alarm pheromone in A. pisum, as well as in M. persicae (Shambaugh et al., 1978; Sun et al., 2012a, 2013). The 6th segment coeloconic pegs were labeled exclusively by antibodies against OBP3 and OBP7 suggesting a role in chemoreception for these uniporous sensilla (Shambaugh et al., 1978; Sun et al., 2013). Their presence in many insect orders and ancient origin (Steinbrecht, 1997) could reveal their critical role and involvement in some basic needs of insects, such as the protection from dangerous conditions. Moreover, our data showed that only OBP6 was expressed in placoid sensilla on the 3rd segment (secondary rhinaria) suggesting a possible involvement into sex pheromone and plant volatile perception (Pickett et al., 1992). Finally, immunohistochemical experiments showed that type I trichoid sensilla were not stained by any anti-OBP antibody, confirming that they could have a mechanoreceptive function as reported in literature (Bromley et al., 1979; Shambaugh, 1978; Sun et al., 2013).

Our data supported the idea that in aphids, different OBPs may colocalize in the same sensilla (Sun et al., 2013). This phenomenon, that have been also observed in other insects (Hekmat-Scafe et al., 1997; Maida et al., 2005; Qiao et al., 2011), can be explained by This article is protected by copyright. All rights reserved. 
considering that aphids have few sensilla, so a single type of olfactory sensillum may respond to a broad spectrum of odorants (Syed \& Leal, 2007) and, in some cases, a single type of sensillum could even play different sensory function, as already reported by Bromley et al. (1980).

Some of the analyzed OBPs were also expressed at higher levels in the terminal region of aphid body. It is the case of OBP1 and OBP3 expressed in trichoid sensilla but also in the caudal digitiform projections, arranged in groups, and in the cornicles, located on the terminal abdomen segments and involved in the secretion of fluid droplets in response to danger, for example the attack by an entomophagous insect (Capinera, 2008). The secretion is composed by the alarm pheromone, that induces conspecific physiological and behavioral responses, such as increased production of winged morph in the offspring and short term defensive responses respectively (Hatano et al., 2010; Kislow \& Edwards, 1972; Kunert et al., 2005; Pickett et al., 1992; Podjasek et al., 2005), as well as other substances, mainly triglycerides (Callow et al., 1973), which seem to act as mechanical defenses by gluing the appendages of natural enemies (Butler \& O’Neil, 2006; Greenway \& Griffiths, 1973; Strong, 1967; van Emden \& Harrington, 2007). Furthermore, at the tip of the abdomen there is a cauda that is well known for having the mechanical function of removing the honeydew droplets produced by aphids (Capinera, 2008) and presents trichoid sensilla and digitiform projections arranged in groups on its surface. In this terminal abdomen region, the expressed OBPs could have different roles; they could act as carriers to the external environment in the releasing of hydrophobic molecules (for example alarm pheromone and triglycerides), rather than being involved in their perception (Calvello et al., 2003; Li et al., 2008; Sun et al., 2012b), and/or OBP1 and OBP3 could be involved in leaf surface probing in recognition of a suitable site for oviposition or giving birth to offspring. 
Moreover, we found that the gene encoding OBP8 was mainly expressed in head and the respective protein immunolocalized in the buccal apparatus especially at the base of the short peg sensilla on the labium tip, where the epithelial cells producing OBPs are located (Pelosi et al., 2006), and in the inner lymph of hair-like sensilla on the labium side walls. It is generally accepted that aphids have no contact chemoreceptors on the exposed parts of the proboscis (Capinera, 2008) and that, although the "probing" activity mediated by chemoreceptors on the epipharynx and hypopharynx plays a major role in host-plant selection (Wensler, 1962; Wensler \& Filshie, 1969), it is also apparent that before probing, plant molecules may influence aphid behavior (Klingauf, 1972; Tjallingii, 1976). Whether or not these plant influences act via gustatory, olfactory, visual, or even mechanoreceptors has not yet been fully clarified. The expression of OBP8 in A. pisum mouthpart sensilla would suggest a possible role in host-plant selection, probably through a mechanism not entirely clarified. On the other hand OBP expression in non-olfactory tissues is an ascertained phenomenon and suggests that they also may function as carriers of chemicals in different developmental and physiological processes (Calvello et al., 2003; Dani et al., 2001; Iovinella et al., 2011; Li et al., 2008; Sun et al., 2012b; Vogel et al., 2010).

Regarding the legs we found a very low expression level for all the analyzed OBPs and a very weak signal in immunolocalization experiments thus, we were not able to make assumptions about the possible role of these polypeptides in chemoreceptive sensilla of these aphid appendages.

Transcription profiling for the OBP genes was also performed for all A. pisum developmental instars including the four pre-reproductive stages and the winged and unwinged adult morph. Aphids exhibit diverse behaviors during different instars; therefore, it is reasonable to expect that their olfactory system might be tuned to different odors in the course of development (Roitberg \& Meyers, 1978). The expression peak for OBP1, OBP3 This article is protected by copyright. All rights reserved. 
and OBP7 in the fourth instar and unwinged adult stage could be due to the involvement of these proteins in the detection of compounds, like $(E)-\beta$-farnesene, particularly important at these stages (Roitberg \& Meyers, 1978; Sun et al., 2012a). In fact, it has been reported that adult and fourth instar pea aphids, compared with younger pre-reproductive instars, exhibit a much stronger response to the alarm pheromone by dropping from the plant, running or backing up. This different behavior has been related to the fact that younger instars are less active on the ground and have less chance of finding their host plant again (Roitberg \& Meyers, 1978). Instead, the lower transcript levels observed in winged adults compared with unwinged adults could be explained for their natural swiftness at moving away from the host plant thanks to the wing presence. On the other hand, it is very important for unwinged aphids to have a strong sensibility to the alarm pheromone perception since it not only induces an escape behavior (Pickett et al., 1992) as short term effect contextually to a dangerous situation, but it also induces a long term physiological response mediating $A$. pisum wing polyphenism (Hatano et al., 2010; Kunert et al., 2005; Podjasek et al., 2005) and thus provides a further survival mechanism for the aphid colony. Indeed, the perception of alarm pheromone causes an increased movement of aphids within the colony increasing the frequency of physical contact, the same condition occurring in crowded populations, with the consequent increased proportion of winged dispersing morph among the progeny (Kunert et $a l ., 2005)$. The relation between the unwinged adult alarm pheromone perception and the wing polyphenism is supported by the evidence provided by Kunert and Weisser (2005) that the antennae have a crucial role in wing induction in offspring and it is relatively linear correlated with the amount and frequency of alarm pheromone release (Kunert et al., 2005; Podjasek et al., 2005). These observations are consistent with our results regarding the presence of OBP3 and OBP7, mainly involved in $(E)-\beta$-farnesene perception (Sun et al., 2012a), in the antennal sensilla. 
The transcript of OBP6 was mainly detected in winged adults, suggesting that it could be involved in the perception of molecules related to new host plant location and sex pheromone perception, furthermore supported by presence of this protein into secondary rhinaria. These rhinaria, more abundant in winged morphs than in apterous aphids, respond to sex pheromone and plant volatiles suggesting their involvement in host location (Pickett et al., 1992). Indeed, it has been reported that the sex pheromone from sexual females serves double purposes and acts also as an aggregation pheromone, attracting asexually reproducing, conspecific winged females (Hardie et al., 1996; Lösel et al., 1996; Park et al., 2000). For gynoparae, this would facilitate in finding a suitable site for producing offspring.

The nearly constant expression level of OBP8 in all aphid instars suggests a basic conserved role of this protein, such as an involvement in feeding, concurring with its presence at the adult buccal apparatus. The higher expression level of OBP8 in winged then in unwinged adults could be related to their important role in the selection of a new host plant (Braendle et al., 2006). On the other side the presence of this protein in non-olfactory tissues or non-sensory organs could be explained with a new or additional function other than odor perception. Anyway understanding of additional or alternative roles outside of olfaction for some of these proteins is a new and important task that deserves further insights.

Overall, OBPs show a very complex expression profile, both relative to body parts and developmental stages, probably in connection with different roles in aphid behavior. Further studies are needed to gain a better understanding of a such significant diversity in their functional role. In particular the biochemical analysis and behavioral studies that allow the association of one or more OBPs to the perception of a specific odor (He et al., 2011; Sun et $a l .$, 2012a) will be supported from the knowledge provided by our study considering that the expression of a protein in a particular cell, tissue or developmental stage is generally motivated by a specific function in that biological context. Any advancement regarding the This article is protected by copyright. All rights reserved. 
decodification at molecular level of the odor perception in aphids will provide insights into determining new strategies for control of these worldwide pests by interfering with their chemical communication.

\section{Acknowledgments}

We would like to thank Prof. Paolo Pelosi (University of Pisa, Italy) for his critical reading of the manuscript and for providing the antibodies against each OBP used in immunolocalization experiments, Luca Trotti for aphid pictures, Dr. Massimo Cristallo for his suggestions on statistical analysis and Dr. Rita Armentano for the English language editing. We thank Dr. Genoveffa Ciancio and Dr. Antonio Romano for their help in insect rearing. We also thank the Interdepartmental Center for Scientific Equipment (CIGAS) of the University of Basilicata, Potenza, for 7500 Fast Real-Time PCR System (Applied Biosystems) availability and the Centro Grandi Attrezzature (CGA) of the University of Insubria for Confocal laser microscope availability. This work was supported by the Scientific Research Fund of the University of Basilicata.

\section{Disclosure}

All authors declare no conflicts of interest.

\section{References}


Blackman, R.L. and Eastop, V.F. (2007) Taxonomic issues. Aphids as Crop Pests (eds. H.F. van Emden \& R. Harrington), pp. 1-29. CAB International, Wallingford.

Braendle, C., Davis, G.K., Brisson, J.A. and Stern, D.L. (2006) Wing dimorphism in aphids. Heredity, 97, 192-199.

Bromley, A.K., Dunn, J.A. and Anderson, M. (1979) Ultrastructure of the antennal sensilla of aphids. I. Coeloconic and placoid sensilla. Cell and Tissue Research, 203, 427-442.

Bromley, A.K., Dunn, J.A. and Anderson, M. (1980) Ultrastructure of the antennal sensilla of aphids. II. Trichoid, chordotonal and campaniform sensilla. Cell and Tissue Research, 205, $493-551$.

Bustin, S.A., Benes, V., Garson, J.A., Hellermans, J., Huggett, J. and Kubista, M. (2009) The MIQE guidelines: minimum information for publication of quantitative real-time PCR Experiments. Clinical Chemistry, 55, 611-622.

Butler, C.D. and O’Neil, R.J. (2006) Defensive response of soybean aphid (Hemiptera: Aphididae) to predation by insidious flower bug (Hemiptera: Anthocoridae). Annals of the Entomological Society of America, 99, 317-320.

Callow, R.K., Greenway, A.R. and Griffiths, D.C. (1973) Chemistry of the secretion from the cornicles of various species of aphids. Journal of Insect Physiology, 19, 737-748.

Calvello, M., Guerra, N., Brandazza, A., D’Ambrosio, C., Scaloni, A., Dani, F.R., Turillazzi, S. and Pelosi, P. (2003) Soluble proteins of chemical communication in the social wasp Polistes dominulus. Cellular and Molecular Life Sciences, 60, 1933-1943.

Capinera, J.L. (2008) Encyclopedia of Entomology. Springer, Dordrecht.

Dani, F.R., Michelucci, E., Francese, S., Mastrobuoni, G., Cappellozza, S., La Marca, G., Niccolini, A., Felicioli, A., Moneti, G. and Pelosi, P. (2001) Odorant-binding proteins and Chemosensory proteins in pheromone detection and release in the silkmoth Bombyx mori. Chemical Senses, 36(4), 335-344. 
Development Core Team (2009) $R$ : A language and environment for statistical computing. $\mathrm{R}$ Foundation for Statistical Computing, Vienna.

Dewhirst, S.Y., Pickett, J.A. and Hardie, J. (2010) Aphid pheromones. Vitamins \& Hormones, 83, 551-574.

Flögel, J.H.L. (1905) Monographie der Johannisbeeren-Blattlaus, Aphis ribis L. Zeitschrift für wissenschaftliche Insektenbiologie, 2, 49-63, 145-155.

Greenway, A.R. and Griffiths, D.C. (1973) A comparison of triglycerides from aphids and their cornicle secretions. Journal of Insect Physiology, 19(8), 1649-1655.

Hansson, B.S. (1999) Insect Olfaction. Springer, Berlin.

Hansson, B.S., Carlsson, M.A. and Kalinovà, B. (2003) Olfactory activation patterns in the antennal lobe of the sphinx moth, Manduca sexta. Journal of Comparative Physiology A. Neuroethology, Sensory, Neural, and Behavioral Physiology, 189, 301-308.

Hardie, J., Storer, J.R., Cook, F.J., Campbell, C.A.M., Wadhams, L.J., Lilley, R. and Peace, L. (1996) Sex pheromone and visual trap interactions in mate location strategies and aggregation by host-alternating aphids in the field. Physiological Entomology, 21, 97-106.

Hatano, E., Kunert, G. and Weisser, W.W. (2010) Aphid wing induction and ecological costs of alarm pheromone emission under field conditions. PLoS ONE, 5: e11188.

He, P., Zhang, J., Liu, N.Y., Zhang, Y.N., Yang, K. and Dong, S.L. (2011) Distinct expression profiles and different functions of Odorant Binding proteins in Nilaparvata lugens Stal. PLoS ONE, 6: e28921.

Hekmat-Scafe, D.S., Steinbrecht, R.A. and Carlson J.R. (1997) Coexpression of two odorantbinding protein homologs in Drosophila: implications for olfactory coding. The Journal of Neuroscience, 17, 1616-1624. 
Hildebrand, J.G. (1996) Olfactory control of behavior in moths: central processing of odor information and the functional significance of olfactory glomeruli. Journal of Comparative Physiology A. Neuroethology, Sensory, Neural, and Behavioral Physiology, 178, 5-19.

Iovinella, I., Dani, F.R., Niccolini, A., Sagona, S., Michelucci, E., Gazzano, A., Turillazzi, S., Felicioli, A. and Pelosi, P. (2011) Differential expression of odorant-binding proteins in the mandibular glands of the honey bee according to caste and age. Journal of Proteomic Research, 10, 3439-3449.

Isidoro, N., Bin, F., Colazza, S. and Vinson, S.B. (1996) Morphology of antennal gustatory sensilla and glands in some parasitoid Hymenoptera with hypothesis on their role in sex and host recognition. Journal of Hymenoptera Research, 5, 206-239.

Kislow, C. and Edwards, L.J. (1972) Repellent odour in aphids. Nature, 235, 108-109.

Klingauf, F., Sengona, C. and Bennewita, H. (1972) Effect of sinigrin on sucrose uptake by some polyphagous and oligophagous aphids Aphididae. Oecologia, 9, 53-57.

Kunert, G., Otto, S., Röse, U.S., Gershenzon, J. and Weisser, W.W. (2005) Alarm pheromone mediates production of winged dispersal morphs in aphids. Ecology Letters, 8, 596-603.

Kunert, G. and Weisser, W.W. (2005) The importance of antennae for pea aphid wing induction in the presence of natural enemies. Bulletin of Entomological Research, 95, 125131.

Laughlin, J.D., Ha, T.S., Jones, D.N.M. and Smith, D.P. (2008) Activation of pheromonesensitive neurons is mediated by conformational activation of pheromone binding protein. Cell, 133, 1255-1265.

Lee, C., Kim, J., Shin, S.G. and Hwang, S. (2006) Absolute and relative QPCR quantification of plasmid copy number in Escherichia coli. Journal of Biotechnology, 123(3), 273-280. 
Li, S., Picimbon, J.F., Ji, S.D., Kan, Y.C., Qiao, C.L., Zhou, J.J. and Pelosi, P. (2008) Multiple functions of an odorant-binding protein in the mosquito Aedes aegypti. Biochemical and Biophysical Research Communications, 372(3), 464-468.

Liu, W. and Saint, D.A. (2002) A new quantitative method of real time reverse transcription polymerase chain reaction assay based on simulation of polymerase chain reaction kinetics. Analytical Biochemistry, 302(1), 52-59.

Lösel, P.M., Lindemann, M., Scherkenbeck, J., Campbell, C.A.M., Hardie, J., Pickett, J.A. and Wadhams, L.J. (1996) Effect of primary-host kairomones on the attractiveness of the hop-aphid sex pheromone to Phorodon humuli males and gynoparae. Entomologia Experimentalis et Applicata, 80, 79-82.

Maida, R., Mameli, M. and Mueller, B. (2005) The expression pattern of four odorantbinding proteins in male and female silk moths, Bombyx mori. Journal of Neurocytology, 34, $149-163$.

Marsh, D. (1975) Responses of male aphids to the female sex pheromone in Megoura viciae Buckton. Journal of Entomology Series A, 50, 43-64.

Matsuo, T., Sugaya, S., Yasukawa, J., Aigaki, T. and Fuyama, Y. (2007) Odorant-binding proteins OBP57d and OBP57e affect taste perception and host-plant preference in Drosophila sechellia. PLoS Biology, 5: e118.

Messchendorp, L., Gols, G. J. Z. and van Loon, J. J. A. (1998) Behavioral effects and sensory detection of drimane deterrents in Myzus persicae and Aphis gossypii nymphs. Journal of Chemical Ecology, 24, 1433-1446.

Moore, R.D., Mumaw, V. and Schoenberg, M.D. (1960) Optical microscopy of ultrathin tissue sections. Journal of Ultrastructure Research, 4, 113-116. 
Park, K.C., Elias, D., Donato, B. and Hardie, J. (2000) Electroantennogram and behavioural responses of different forms of the bird cherry-oat aphid, Rhopalosiphum padi, to sex pheromone and a plant volatile. Journal of Insect Physiology, 46, 597-604.

Pelosi, P., Zhou, J.J., Ban, L.P. and Calvello, M. (2006) Soluble proteins in insect chemical communication. Cellular and Molecular Life Sciences, 63, 1658-1676.

Pettersson, J. (1971) An aphid sex attractant. II. Histological, ethological and comparative studies. Entomologica Scandinavica, 2, 81-93.

Pettersson, J., Tjallingii, W.F. and Hardie, J. (2007) Host-plant Selection and Feeding. Aphids as Crop Pests (eds. H.F. van Emden \& R. Harrington), pp. 87-113. CAB International, Wallingford.

Pickett, J.A., Wadhams, L.J., Woodcock, C.M. and Hardie, J. (1992) The chemical ecology of aphids. Annual Review of Entomology, 37, 67-90.

Plettner, E. (2002) Insect pheromone olfaction: new targets for the design of species-selective pest control agents. Current Medicinal Chemistry, 9, 1075-1085.

Podjasek, J.O., Bosnjak, L.M., Brooker, D.J. and Mondor, E.B. (2005) Alarm pheromone induces a transgenerational wing polyphenism in the pea aphid, Acyrthosiphon pisum. Canadian Journal of Zoology, 83, 1138-1141.

Qiao, H.L., He, X.L., Schymura, D., Ban, L.P., Field, L., Dani, F.R., Michelucci, E., Caputo, B., della Torre, A., Iatrou K., Zhou, J.J., Krieger, J. and Pelosi P. (2011) Cooperative interactions between odorant-binding proteins of Anopheles gambiae. Cellular and Molecular Life Sciences, 68, 1799-1813.

Roitberg, B.D. and Meyers, J.H. (1978) Adaptation of alarm pheromone responses of the pea aphid Acyrthosiphon pisum (Harris). Canadian Journal of Zoology, 56(1), 103-108.

Romani, R. and Rossi Stacconi, M.V. (2009) Mapping and ultrastructure of antennal chemosensilla of the wheat bug Eurygaster maura. Insect Science, 16, 193-203. 
Ryan, M.F. (2002) Insect chemoreception: fundamental and applied. Kluwer Academic Publishers, Dordrecht.

Shambaugh, G.F., Frazier J.L., Castell, A.E.M. and Coons, L.B. (1978) Antennal sensilla of seventeen aphid species (Homoptera:Aphidinae). International Journal of Insect Morphology and Embryology, 7, 389-404.

Shields, V.D.C. and Hildebrand, J.G. (2001) Recent advances in insect olfaction, specifically regarding the morphology and sensory physiology of antennal sensilla of the female sphinx moth Manduca sexta. Microscopy Research and Technique, 55(5), 307-329.

Steinbrecht, R.A. (1997) Pore structures in insect olfactory sensilla: a review of data and concepts. International Journal of Insect Morphology and Embryology, 26, 29 -245.

Strong, F.E. (1967) Observations on aphid cornicle secretions. Annals of the Entomological Society of America, 60, 668-673.

Sun, Y., Qiao, H., Ling, Y., Yang, S., Rui, C., Pelosi, P. and Yang, X. (2011) New analogues of $(E)$ - $\beta$-farnesene with insecticidal activity and binding affinity to aphid odorant-binding proteins. Journal of Agricultural and Food Chemistry, 59, 2456-2461.

Sun, Y.F., De Biasio, F., Qiao, H.L., Iovinella, I., Yang, S.X., Ling, Y., Riviello, L., Battaglia, D., Falabella, P., Yang, X.L. and Pelosi, P. (2012a) Two odorant-binding proteins mediate the behavioural response of aphids to the alarm pheromone $(E)-\beta$-farnesene and structural analogues. PLoS ONE, 7: e32759.

Sun, Y.L., Huang, L.Q., Pelosi, P. and Wang, C-Z. (2012b) Expression in antennae and reproductive organs suggests a dual role of an odorant-binding protein in two sibling Helicoverpa species. PLoS ONE, 7: e30040.

Sun, Y.P., Zhao, L.J., Sun, L., Zhang, S.G. and Ban, L.P. (2013) Immunolocalization of odorant-binding proteins on antennal chemosensilla of the peach aphid Myzus persicae (Sulzer). Chemical Senses, 38(2), 129-136. 
Swarup, S., Williams, T.I. and Anholt, R.R. (2011) Functional dissection of Odorant binding protein genes in Drosophila melanogaster. Genes, Brain and Behavior, 10, 648-657.

Syed, Z. and Leal, W.S. (2007) Maxillary palps are broad spectrum odorant detectors in Culex quinquefasciatus. Chemical Senses, 32, 727-738.

Tjallingii, W.F. (1976) A preliminary study of host selection and acceptance behaviour in the cabbage aphid, Brevicoryne brassicae L. Symposium Biologica Hungarica, 16, 283-285.

Tjallingii, W.F. (1978) Mechanoreceptors of the aphid labium. Entomologia experimentlis et applicata, 24, 531-537.

Van Emden, H.F. and Harrington, R. (2007) Aphids As Crop Pests. CAB International, Wallingford.

Van Giessen, W.A., Fescemyer, H.W., Burrows, P.M., Peterson, J.K. and Barnett, O.W. (1994) Quantification of electroantennogram responses of the primary rhinaria of Acyrthosiphon pisum (Harris) to $\mathrm{C} 4-\mathrm{C} 8$ primary alcohols and aldehydes. Journal of Chemical Ecology, 20(4), 909-927.

Vogel, H., Heidel, A.J., Heckel, D.G. and Groot, A.T. (2010) Transcriptome analysis of the sex pheromone gland of the noctuid moth Heliothis virescens. BMC Genomics, 11, 29.

Vogt, R.G. (2003) Biochemical diversity of odor detection: OBPs, ODEs and SNMPs. Insect Pheromone Biochemistry and Molecular Biology (eds. G.J. Blomquist \& R.G. Vogt), pp. 391-446. Elsevier Academic Press, London.

Wensler, R.J.D. (1962) Mode of host selection by an aphid. Nature, 195, 830-831.

Wensler, R.J.D. (1977) The fine structure of distal receptors on the labium of the aphid, Brevicoryne brassicae L. (Homoptera). Cell and Tissue Research, 181, 409-422.

Wensler, R.J.D. and Filshie B.K. (1969) Gustatory sense organs in the food canal of aphids. Journal of Morphology, 129(4), 473-492. 
Wohlers, P. and Tjallingii, WF. (1983) Electroantennogram responses of aphids to the alarm pheromone E- $\beta$-farnesene. Entomologia Experimentalis et Applicata, 331, 79-82.

Xu, P., Atkinson, R., Jones, D.N. and Smith, D.P. (2005) Drosophila OBP LUSH is required for activity of pheromone-sensitive neurons. Neuron, 45, 193-200.

Zhou, J.J, Field, L.M. and He, X.L. (2010a) Insect odorant-binding proteins: do they offer an alternative pest control strategy? Outlooks on Pest Manage, 21, 31-34.

Zhou, J.J., Vieira, F.G., He, X.L, Smadja, C., Liu, R., Rozas, J. and Field, L.M. (2010b)

Genome annotation and comparative analyses of the odorant-binding proteins and chemosensory proteins in the pea aphid Acyrthosiphon pisum. Insect Molecular Biology, 19 (Suppl. 2), 113-122.

Zhu, J., Zhang, A., Park, K.C., Baker, T., Lang, B., Jurenka, R., Obrycki, J.J., Graves, W.R., Pickett, J.A., Smiley, D., Chauhan, K.R. and Klun, J.A. (2006) Sex pheromone of the soybean aphid, Aphis glycines Matsumura, and its potential use in semiochemical-based control. Environmental Entomology, 35(2), 249-257.

Accepted February 18, 2014 
Fig. 2 SEM images showing the distribution and morphology of different sensilla on A. pisum antennae. A-C: details of type II trichoid sensilla located on the processus terminalis (A) and on the tip of antenna (B) with a single apical pore (arrowhead in C). D-G: details of the primary rhinaria on 6th segment constituted by one large placoid sensillum (LP), two small placoid sensilla (SP) and four coeloconic pegs (CI and CII) all surrounded by a fringed cuticular ridge (arrowheads in E-G). F, G: an enlarged view of coeloconic sensilla of type I (F) and type II (G). H, I: detail of placoid sensillum (arrows) on 5th segment surrounded by a fringed cuticular ridge (arrowheads I). J-L: 3rd segment placoid sensilla (secondary rhinaria) (arrowheads in J, L) and type I trichoid sensilla (arrows) with a rounded poreless tip (K). 


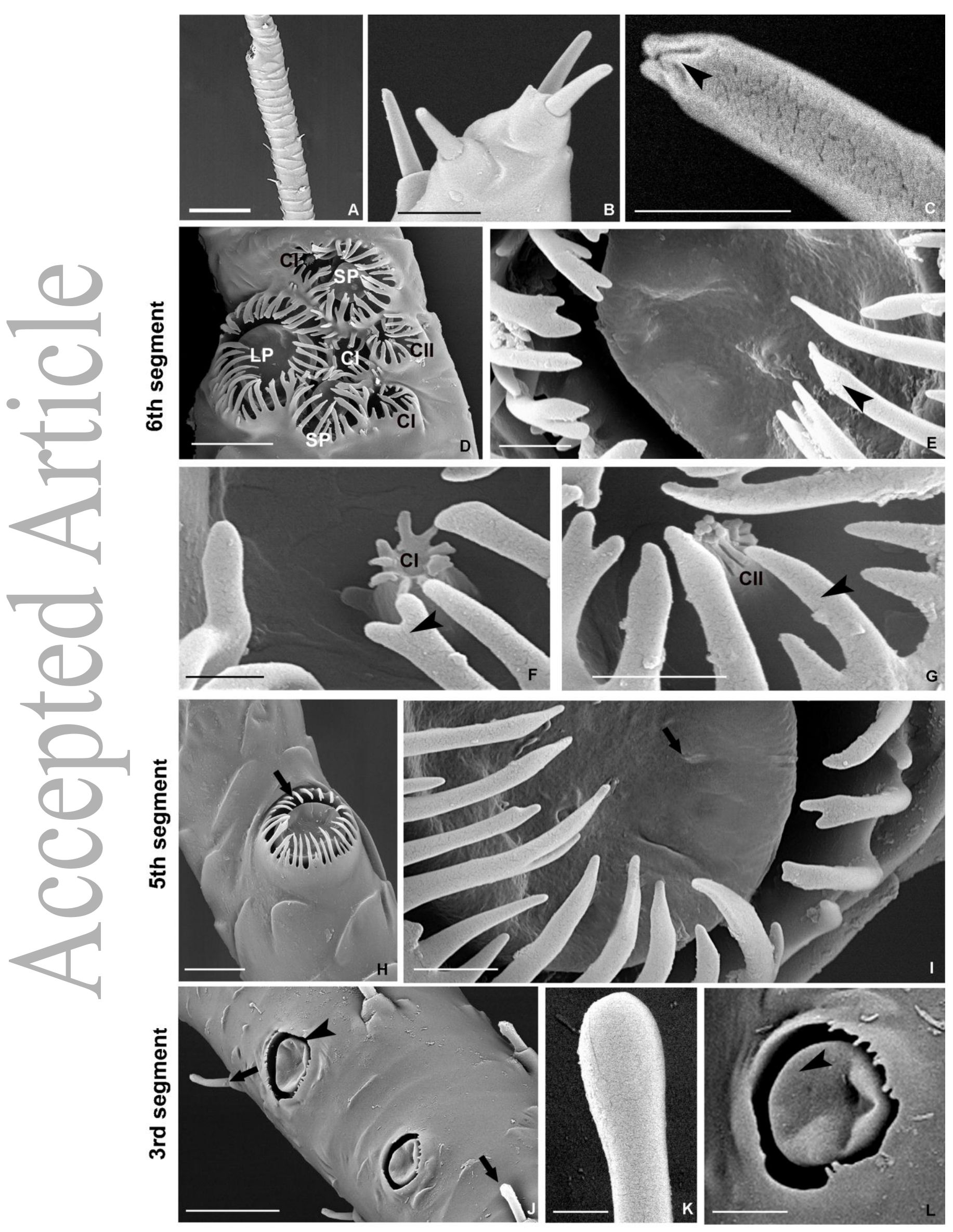

This article is protected by copyright. All rights reserved. 


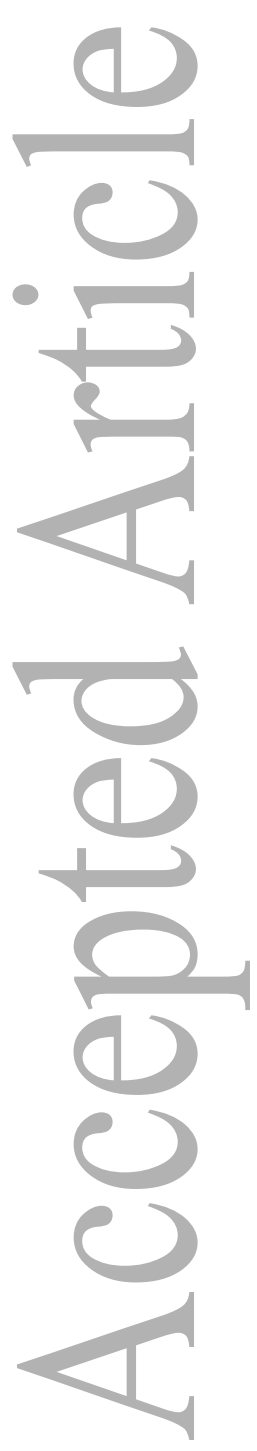


Fig. 3 Dorsal view of A. pisum labium at SEM. A: hair-like structures symmetrically distributed (white arrowheads) and 16 short sensilla (black arrowheads) are visible on the distal region of labium. B: detail of the long hair tip. A fissure-like structure is recognizable (black arrowhead). C: detail of the pore-like structures (molting pores) located at the base of short sensilla (black arrowhead). D, E: SEM detail of long hairs (black arrowhead in D) and digitiform projections (black arrowhead in E) localized on cauda. Bar in A, $20 \mu \mathrm{m}$; bar in B, $500 \mathrm{~nm}$; bar in C, $2 \mu \mathrm{m}$; bar in D $50 \mu \mathrm{m}$; bar in E, $5 \mu \mathrm{m}$.

\section{BUCCAL APPARATUS}
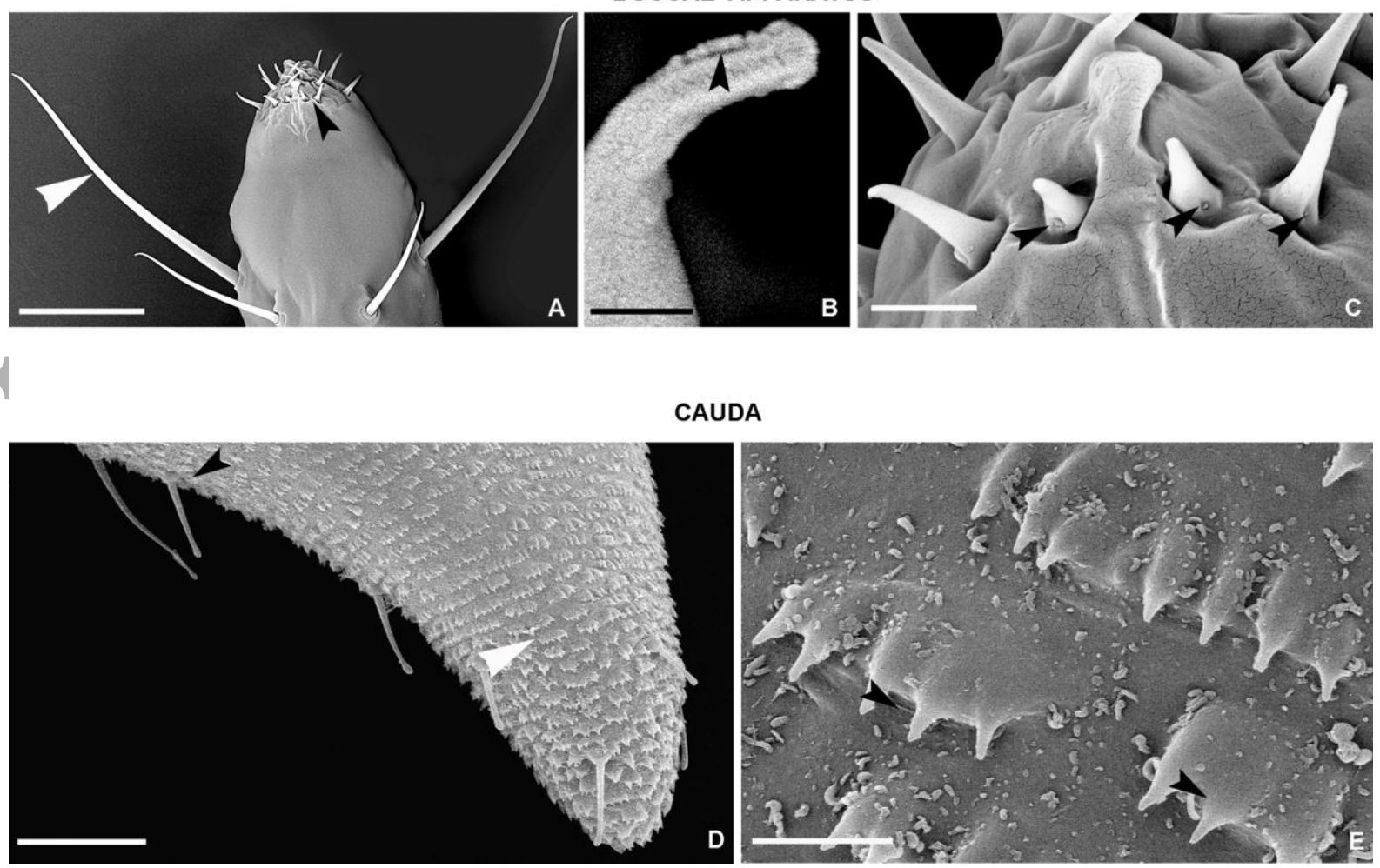
Fig. 4 Relative expression level of $A$. pisum OBPs in different body parts. OBP expression levels were quantified by qRT-PCR. Error bars represent the standard error of the mean for three independent experiments. Significant differences are denoted by different letters (Tukey's test, $P<0.05)$. Lg: legs; Cd: cornicles-cauda; Hd: head; Bd: body; An: antennae. Reference gene: Actin. Calibrator sample: legs. 

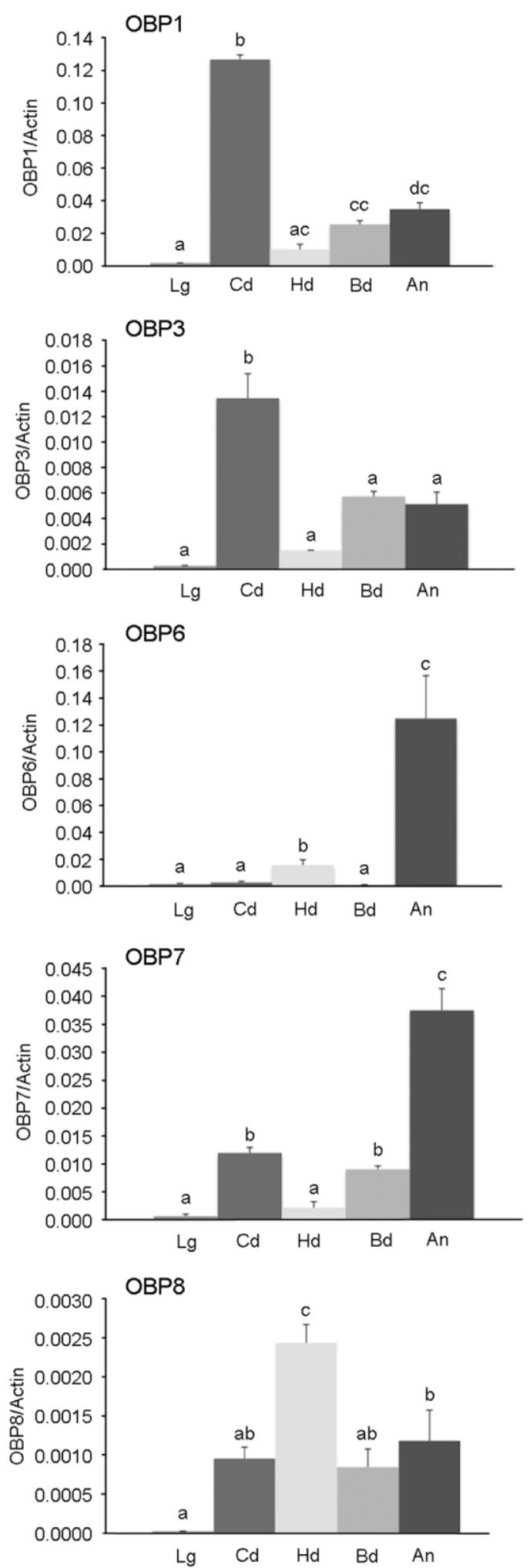
Fig. 5 Whole mount immunolocalization experiments showing the different OBP expression in type II trichoid sensilla located on the antennal tip (A-C) and along the 6th segment $(\mathrm{E}-$ $\mathrm{G})$, in primary rhinaria on 6th $(\mathrm{I}-\mathrm{K})$ and 5th segment $(\mathrm{M}-\mathrm{O})$ and on placoid sensilla of 3rd segment (secondary rhinaria) (Q-S). D, H, L, P, T: negative control. Bars in A-D, $5 \mu \mathrm{m}$; bars in $\mathrm{I}-\mathrm{P}, 10 \mu \mathrm{m}$; bars in $\mathrm{Q}-\mathrm{T}, 25 \mu \mathrm{m}$. 
Fig. 6 Whole mount immunolocalization experiments showing the OBP expression in the terminal body part (arrowheads in A and B) and buccal apparatus (arrowhead in O) sensilla. C-E: immunolocalization in the cornicles. F-N: immunolocalization in the hair-like structures and cauda digitiform projections. P, Q: immunolocalization in the long $(\mathrm{P})$ and short sensilla (Q) located on the distal part of labium. E, L, M, N, R: negative control. Bars in B-E, $50 \mu \mathrm{m}$; bars in F, I, L, $25 \mu \mathrm{m}$; bars in G, H, J, K, M, N, $10 \mu \mathrm{m}$; bars in P, Q, $10 \mu \mathrm{m}$; bar in $\mathrm{R}, 25 \mu \mathrm{m}$. 


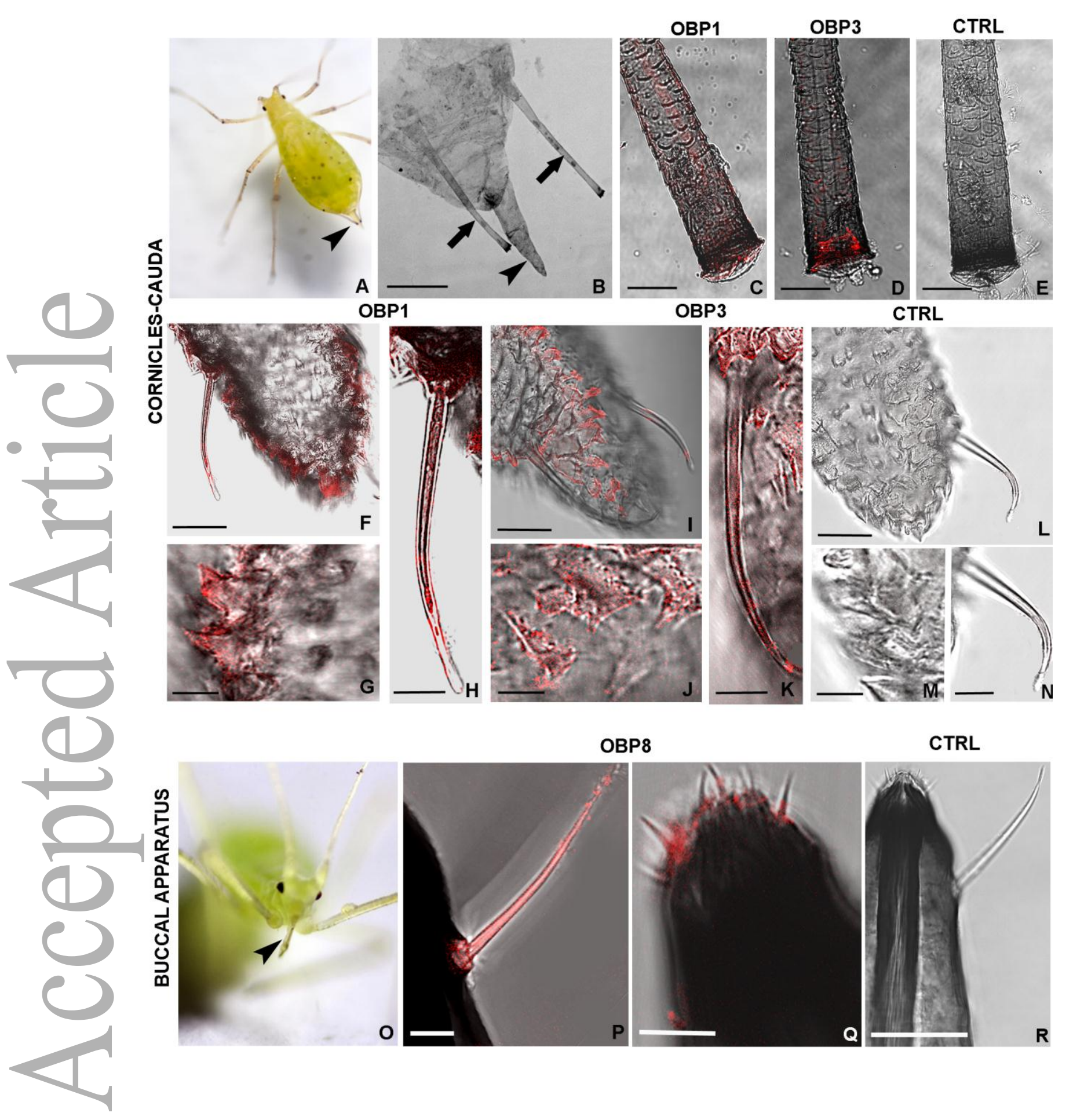


Fig. 7 Relative expression level of A. pisum OBPs in different instars. OBP expression levels were quantified by qRT-PCR. Error bars represent the standard error of the mean for three independent experiments. Significant differences are denoted by different letters (Tukey's test, $P<0.05)$. I: 1 st instar; II: 2nd instar; III: 3rd instar; IV: 4th instar; Ap: apterous adults; Al: alata adults. Reference gene: Actin. Calibrator sample: I: 1st instar. 

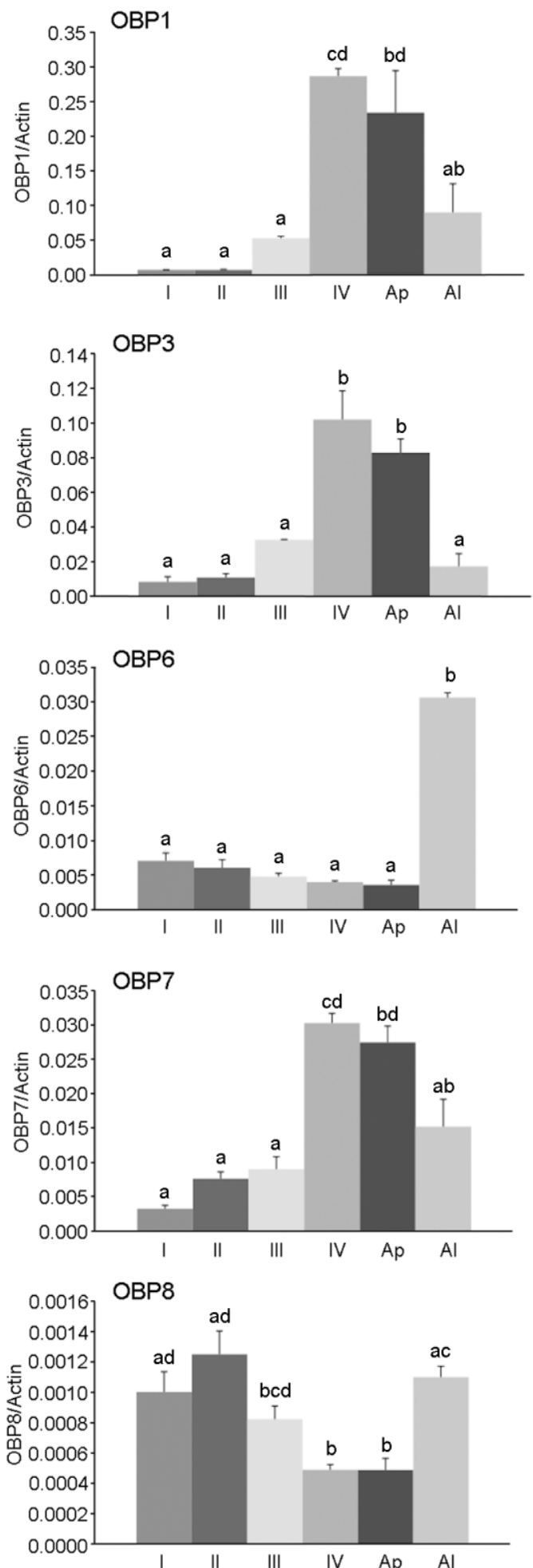
Table 1 Primers used for quantitative RT-PCR.

\begin{tabular}{ccccc}
\hline $\begin{array}{c}\text { Gene } \\
\text { name }\end{array}$ & $\begin{array}{c}\text { Reference sequence } \\
\text { accession }\end{array}$ & \multicolumn{1}{c}{ Primer sequence $\left(5^{\prime}-3^{\prime}\right)$} & $\begin{array}{c}\text { Annealing } \\
\text { temperature } \\
\left({ }^{\circ} \mathrm{C}\right)\end{array}$ & $\begin{array}{c}\text { Amplicon } \\
\text { size (bp) }\end{array}$ \\
\hline OBP1 & NM_001160054.1 & F: GGCAGTAGAGAGTTGTCTATTGGAAA & 58 & 116 \\
& & R: TACATTTATGGGCATGCGACTT & 58 & 77 \\
OBP3 & NM_001160057.1 & F: GGAGCAAATCGATTATTATGGAAAA & 59 & 92 \\
& & R: GCACCTTGTAGGATTTGACTACGA & 58 & 68 \\
OBP6 & NM_001160060.1 & F: ATCATGAATACTCCAGCAGG & 58 & 71 \\
& & R: TCTGAGCTTTCAACCCATAC & 60 & \\
OBP7 & NM_001160061.1 & F: GCCCGGAAAAGAATGTATAACATG & 60 & \\
& & R: AAGACGGTCGCCGCTATG & 58 & 70 \\
OBP8 & NM_001160062.1 & F: AAAAGGCAAAAGACCTCATCGA & 59 & \\
& & R: GGCCAACGCACACTCTTCA & 59 & \\
Actin & NM_001142636.1 & F: CAGATGTGGATCTCCAAACAAGAA & 59 & 58 \\
& & R: CTTAGAAGCATTTACGGTGGACAA & 58 &
\end{tabular}

F, forward primer; R, reverse primer; Actin, reference gene. 